\title{
Online b-tagging selection for the ATLAS experiment at the LHC
}

Andreas Kootz ${ }^{1}$, Jenny Böhme ${ }^{1}$, Mario Cervetto ${ }^{2}$ Peter Mättig ${ }^{1}$, Fabrizio Parodi $^{2}$, Carlo Schiavi $^{2}$, Anca Siebel $^{1}$

${ }^{1}$ Bergische Universität Wuppertal - ${ }^{2}$ Universita degli Studi di Genova Dipart. di Fisica - ATLAS High Level Trigger (HLT) group

Many interesting events in the ATLAS experiment will contain b-jets, for example Higgs or $\mathrm{t} \bar{t}$ production. The LHC will produce $10^{6}-10^{7} \mathrm{t} \mathfrak{t}$ pairs produced per year. The task of the trigger is to select as many as possible to ensure small backgrounds. The background, mainly consists of multijet QCD events, which are predominaltly light quark jets. These jets are almost lightquark jets.

There are several ways for identifying B-hadrons. B-hadrons can be identified for example due to their relative long lifetime. Algorithms making use of the lifetime are using the transverse impact parameter distribution of the tracks or search for secondary vertices. Other algorithms make use of i.e. the jet shape or a soft lepton in a jet, which is also an indicator of a b-quark jet.

\section{ATLAS Trigger System}

The ATLAS Trigger System will be contain three levels. Its task is to select interesting events and thus decrease the incoming data rate from $40 \mathrm{MHz}$ to at most $100 \mathrm{~Hz}$.

The Level 1 trigger will use information from the caloriemeters and from the muon chambers. This level is realized in hardware. It will reduce the data rate to $\sim 100 \mathrm{kKz}$ in a maximal latency of $2.5 \mu \mathrm{s}$

The Level 2 trigger is realized in software. It is running special algorithms. While it will get information from all detectors it works only inside a Region of Interest (RoI) given by the first level trigger. This has to be done to keep time consumption small. The Level 2 trigger will reduce the rate to $\sim 1 \mathrm{kHz}$ in an average time of $10 \mathrm{~ms}$.

The third level trigger - the Event Filter - creates its decision based on the whole event This software filter will reduce the rate to the required one of $\sim 100 \mathrm{~Hz}$ in at most a few seconds. For this it will use software used also in the offline analyses.

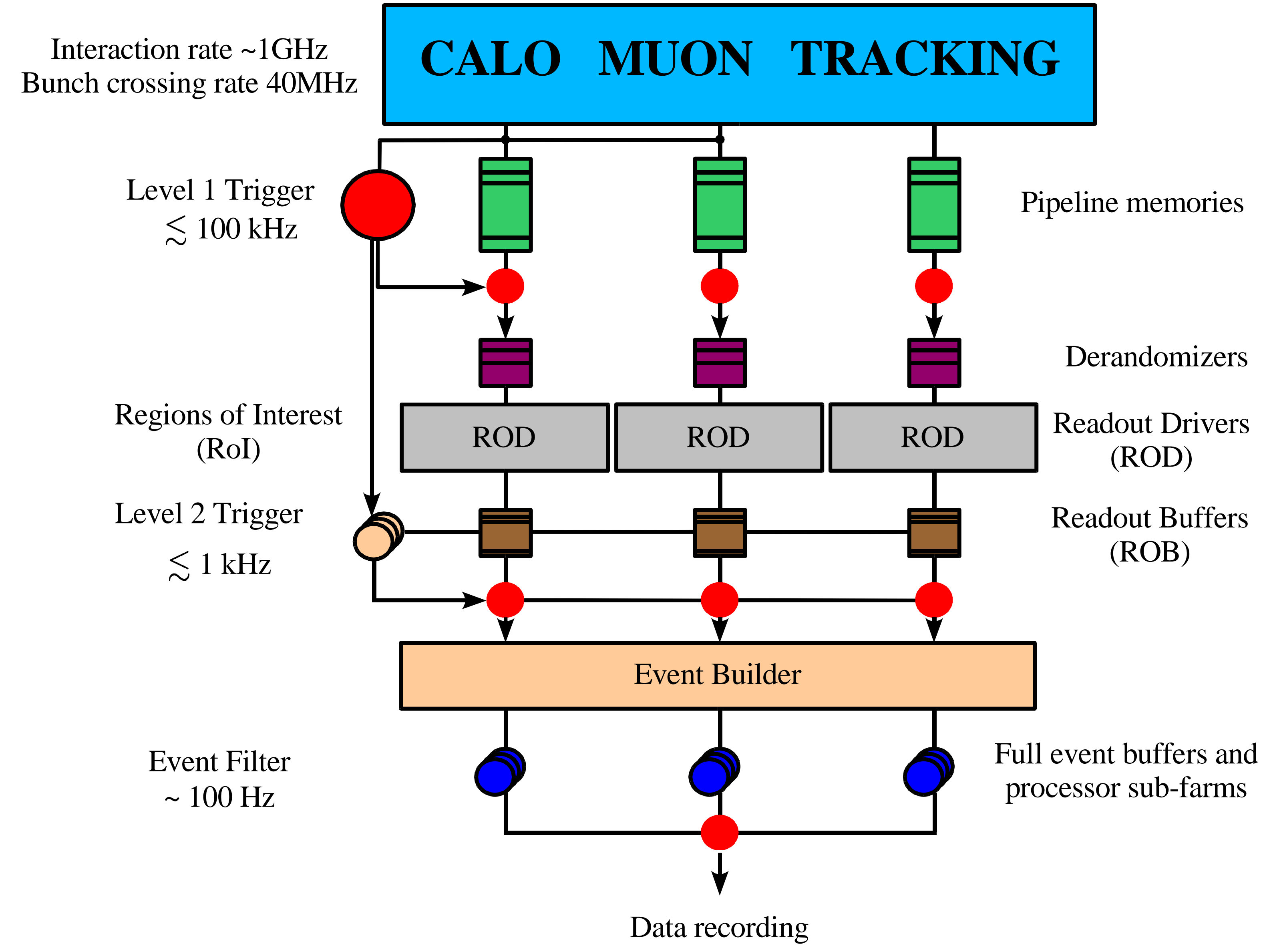

\section{b-jet selection using impact parameter}

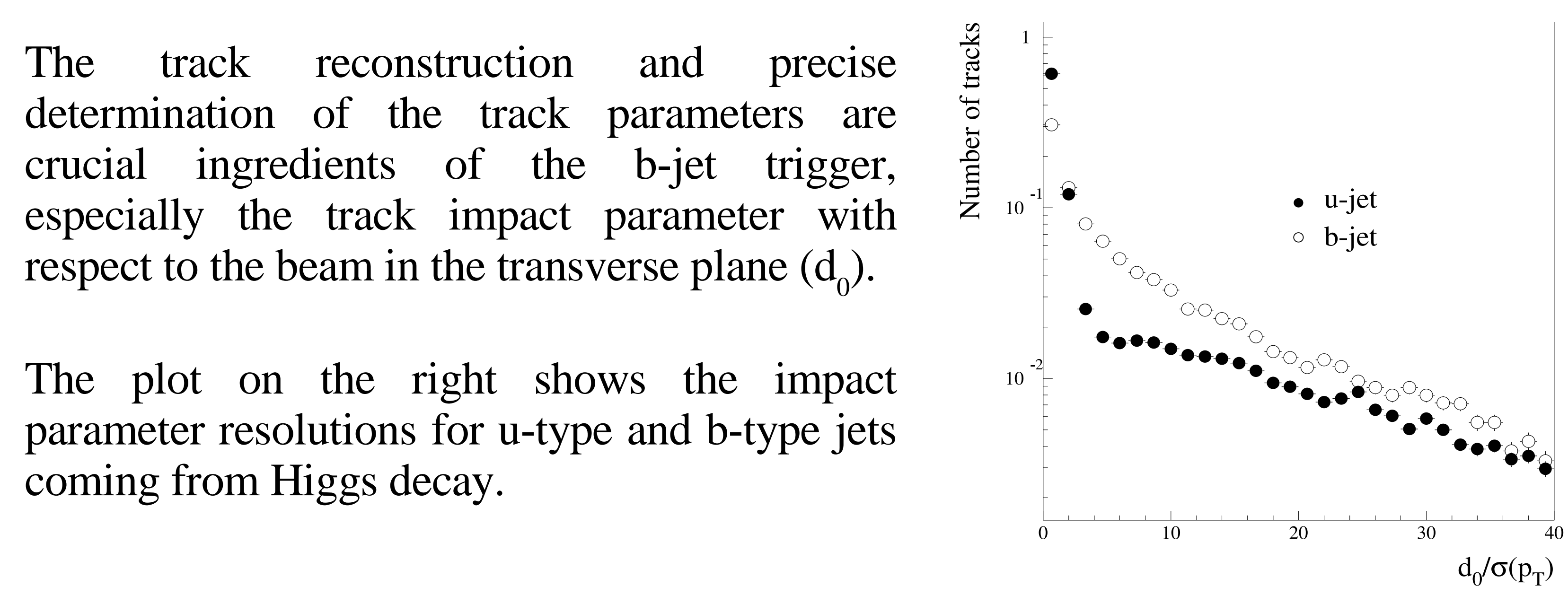

One implementation of the b-jet selection is based on SiTrack, a fast tracking algorithm based on Inner Silicon Detectors that selects and builds track candidates using triplets of space points from the innermost layers granting good $\mathrm{d}_{0}$ resolution.

For each reconstructed track the probability density for a track to come from a b-jet or u-jet is calculated: $\mathrm{P}=\mathrm{f}_{\mathrm{b}}(\mathrm{S}) / \mathrm{f}_{\mathrm{u}}(\mathrm{S})$ with $\mathrm{S}=\mathrm{d}_{0} / \sigma\left(\mathrm{d}_{0}\right)$ being the significance of the track. $\sigma$ is the resolution of the impact parameter. The final b-jet estimator $\mathrm{X}$ is built applying the likelihood-ratio method on the distribution of the reconstructed $\mathrm{d}_{0}: \mathrm{X}=\mathrm{W} /(1+\mathrm{W})$ with $\mathrm{W}$ being the product of the probability densities of all tracks.

The selection has been tested on simulated b-jets and $\mathrm{u}$-jets coming from $\mathrm{H}$ decays produced in association with a $\mathrm{W}$, showing good performance in term of u-rejection and b-efficiency with very little dependence on the luminosity and track density.

The timing performance are compatible with the LVL2 requirements.
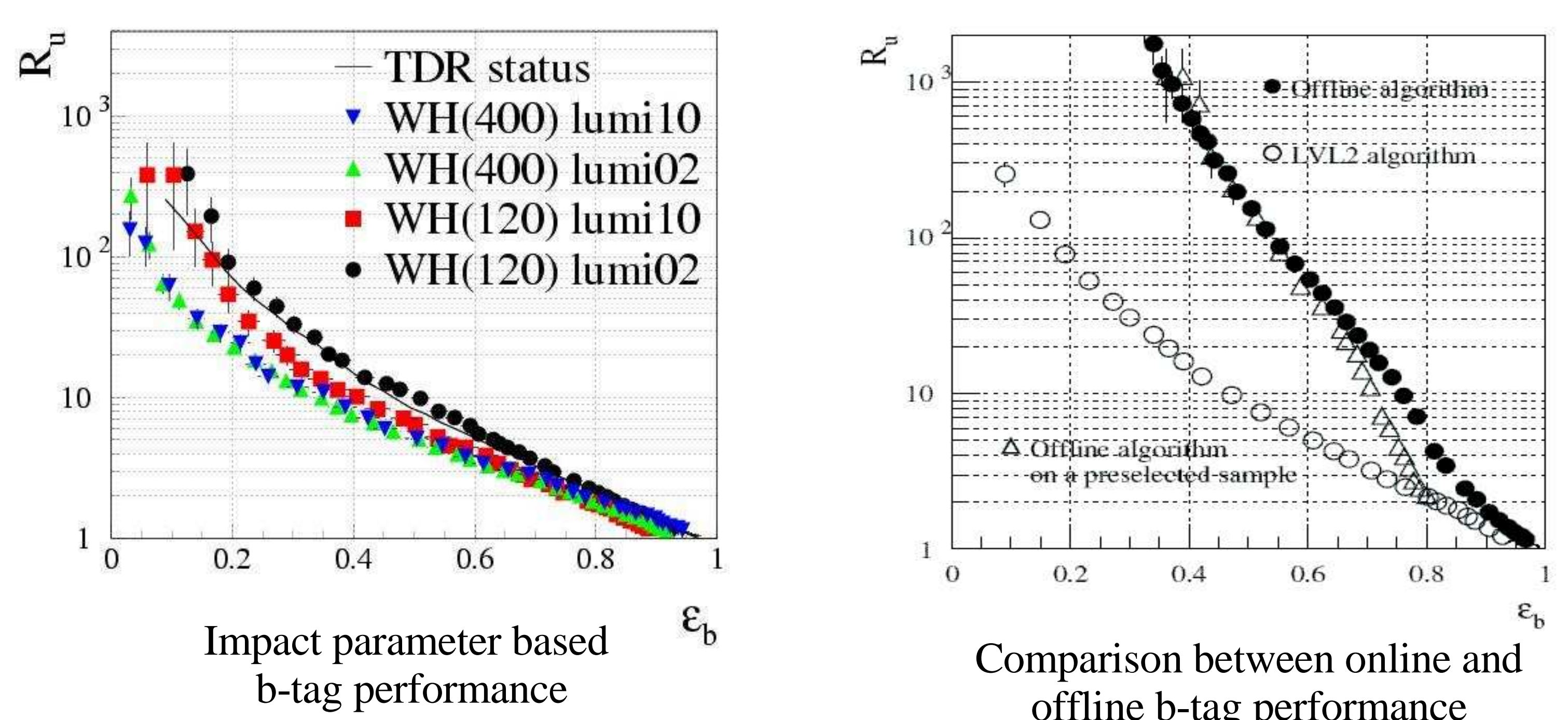

\section{Jet Trigger}

The b-tag algorithm will be called for jet RoIs. The ATLAS trigger system will have settings for 1,2, 3 and 4 jets with different thresholds:

\begin{tabular}{|l|l|l|}
\hline & Level 1 & Level 2 \\
\hline 1 Jet with & $>200 \mathrm{GeV}$ & $>400 \mathrm{GeV}$ \\
\hline 2 Jets with & $>170 \mathrm{GeV}$ & $>350 \mathrm{GeV}$ \\
\hline 3 Jets with & $>90 \mathrm{GeV}$ & $>165 \mathrm{GeV}$ \\
\hline 4 Jets with & $>65 \mathrm{GeV}$ & $>110 \mathrm{GeV}$ \\
\hline
\end{tabular}

With all these triggers the efficiency to select fully hadronic $t \bar{t}$ events is about $1.5-2 \%$ while the purity is $\sim 0.01 \%$. The possibility of selecting b-quark jets at Level 2 should decrease these thresholds without increasing the total rate.

The following two plots show the performance of the 4 Jets selection at the second level trigger as a function of the 4 Jet threshold. The plot on the left shows the behaviour with no required $b$-tag, while the right one shows the behaviour with one b-tag. The curves are normalized to the according values with no b-tag and a trigger threshold of $110 \mathrm{GeV}$. The plots show an increase of efficiency times purity when lowering the threshold to the Level 14 -Jet trigger threshold of $65 \mathrm{GeV}$. When applying b-tagging, the number of triggered events does only exceed 1 at thresholds below $\sim 40 \mathrm{GeV}$.

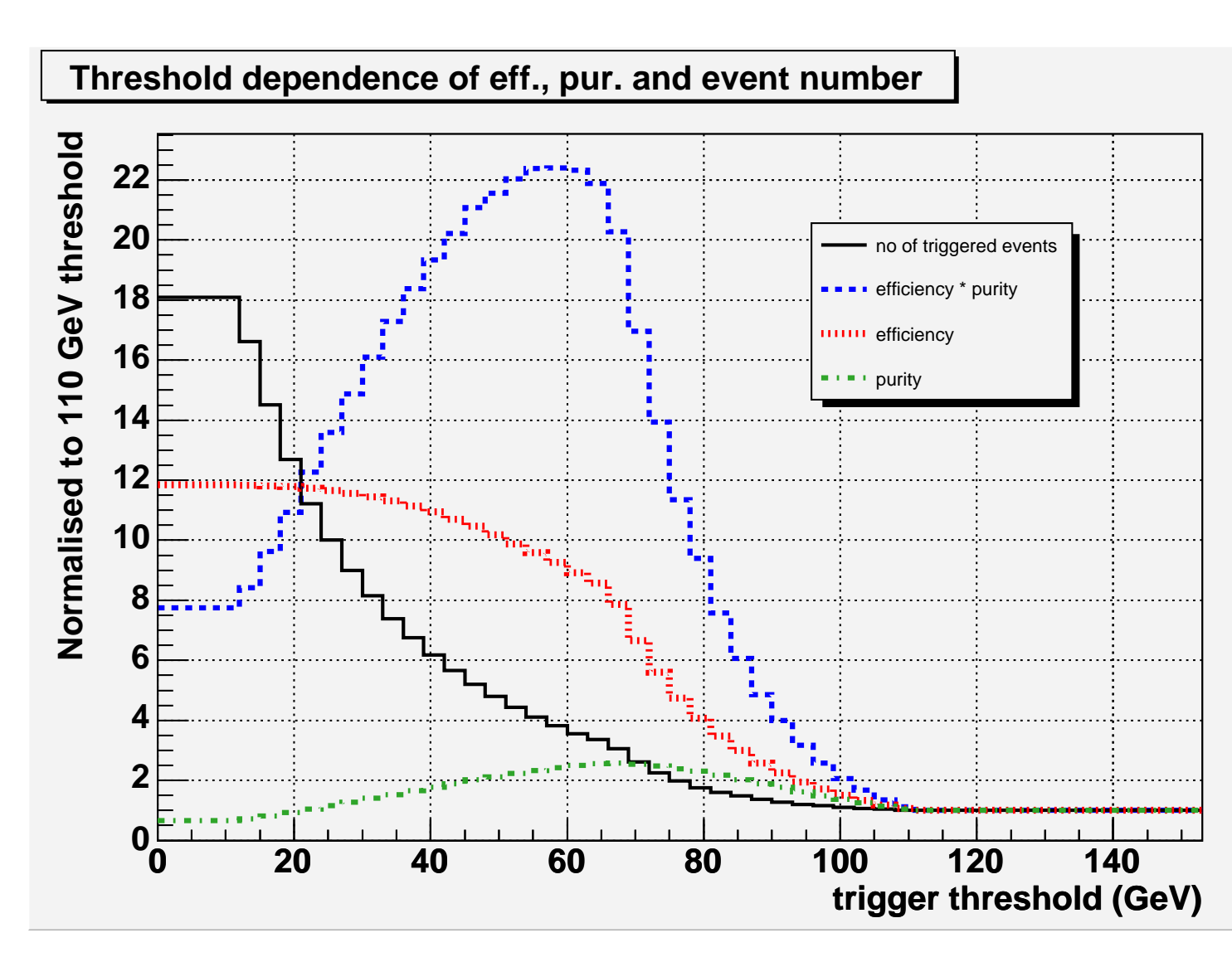

Trigger performance when reducing Level 2 threshold and require $1 \mathrm{~b}$-jet being tagged

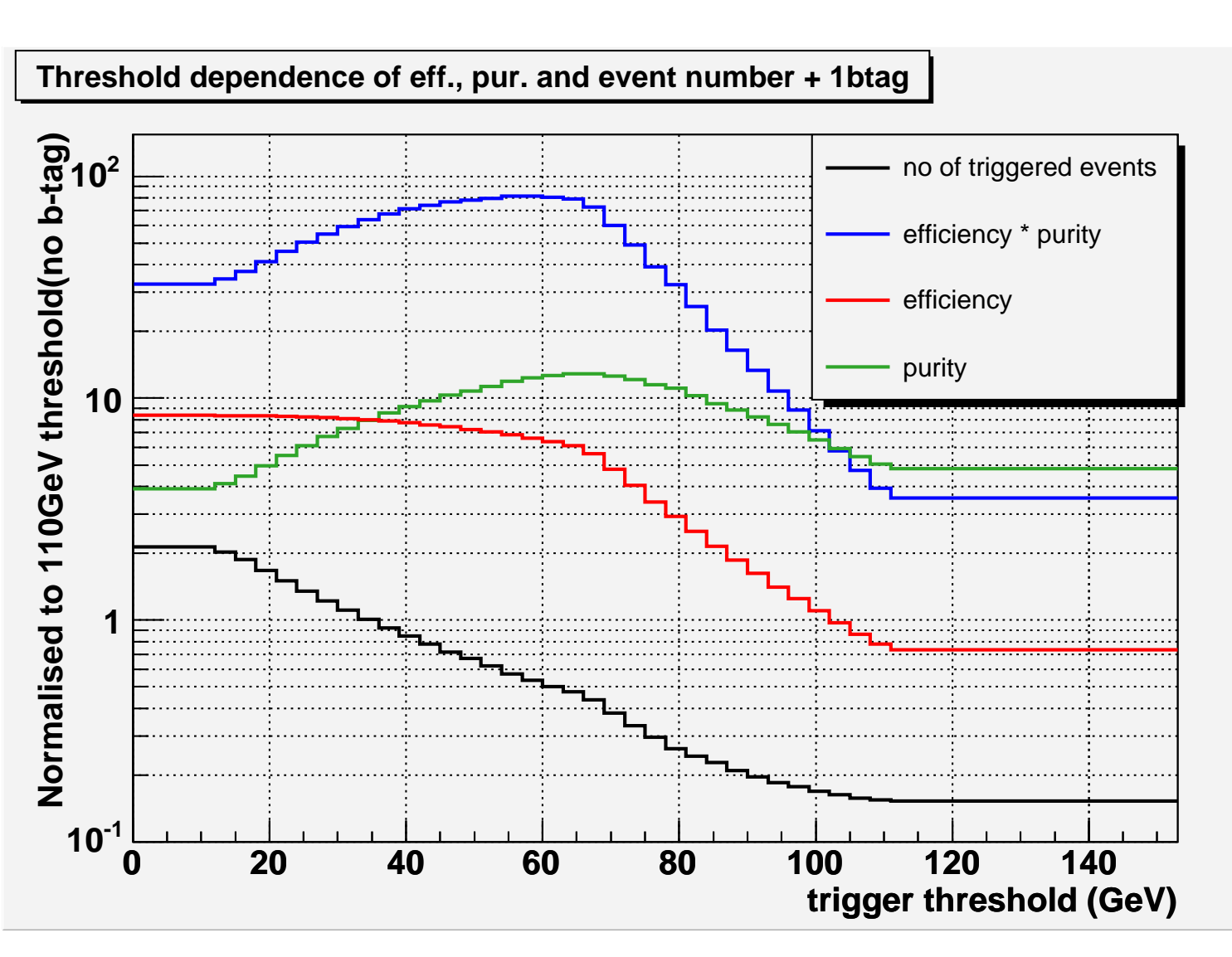

Trigger performance when reducing Level 2 threshold and require $2 \mathrm{~b}$-jets being tagged

\section{b-jet selection using secondary vertices}

The possibility to identify b-quark jets by a secondary vertex was examined with a fast vertex algorithm using perigee parametrization for the tracks. To keep the algorithm fast the method sets three of the five parameters constant. Additionally it is assumed that the track parameter are linearly related to small variations of the vertex or the track momenta.

The method searches for the three dimensional primary and secondary vertices with different subsamples of the tracks in the jet. To get the algorithm fast enough for the use in the ATLAS trigger system, the subsamples have been setup in just one step. Tracks can be deleted in the search but it is not foreseen to add new tracks to the samples.

The b-tag decision is based on the distance between the primary and secondary vertex. Other variables, like the invariant vertex mass, the number of tracks fitted into the secondary vertex and the number of tracks in the jet-RoI are also integrated in the method.

The used regions of interest in that study had all an energy of more than $65 \mathrm{GeV}$ (Level 14 -Jet threshold). A secondary vertex was found in $\sim 60 \%$ of all these regions of interests.

The performance of this algorithm was studied using $t \bar{t}$ events. As a top-quark decays into a $\mathrm{W}$ and a b-quark, this sample contains $2 \mathrm{~b}$-jets per event. The $\mathrm{W}$ decays into a $\mathrm{q} \overline{\mathrm{q}}$ pair or a lepton-neutrino pair. The quarks from the $\mathrm{W}$ decay are mainly light quarks. Thus they can be used to determine the reduction rate against light quark jets.

To study the performance of the algorithm without including performance of the tracking algorithm smeared simulated tracks have been used instead of reconstructed ones.

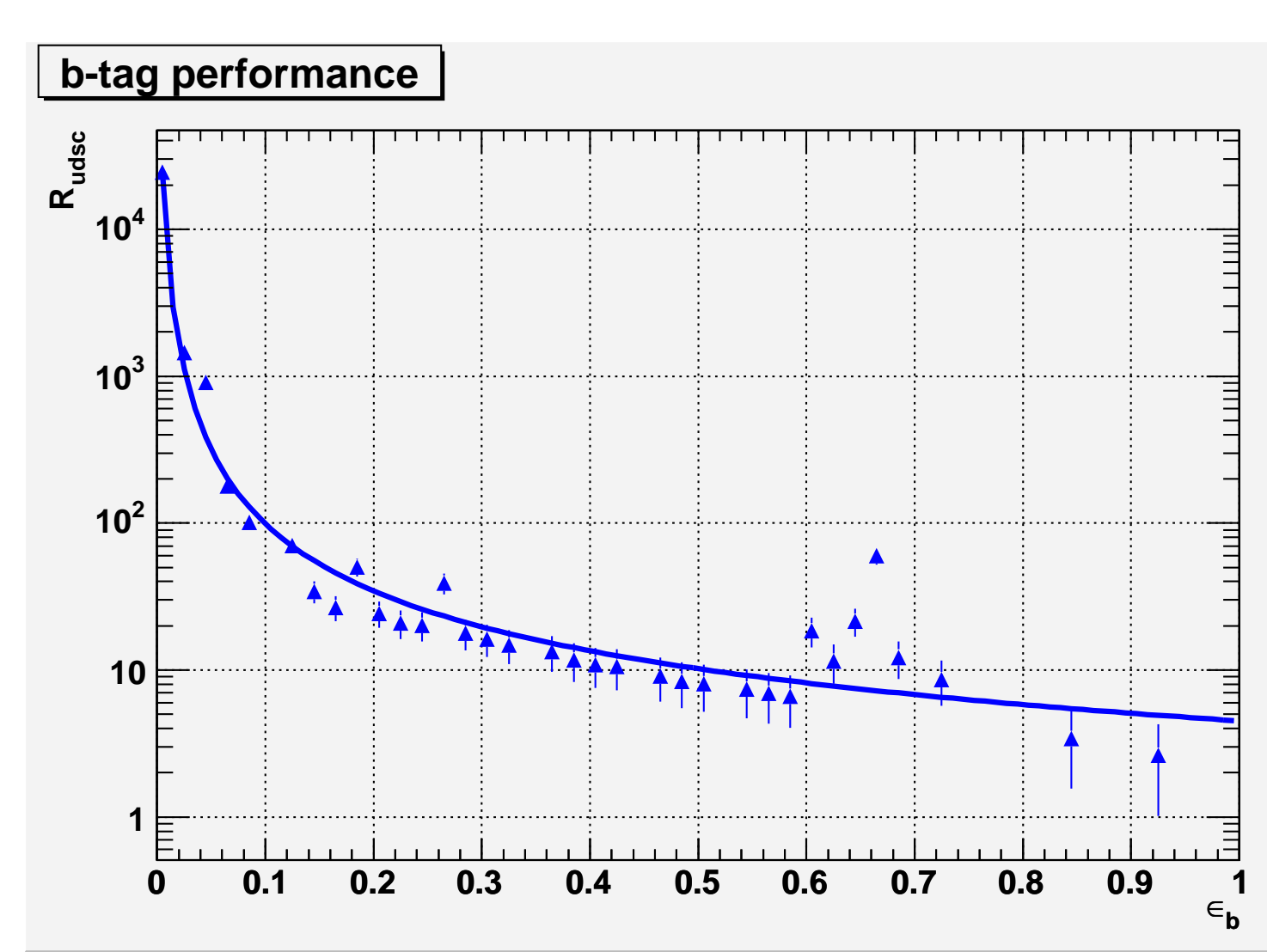

Performance of the vertex based b-tag algorithm based on smeared simulated tracks.

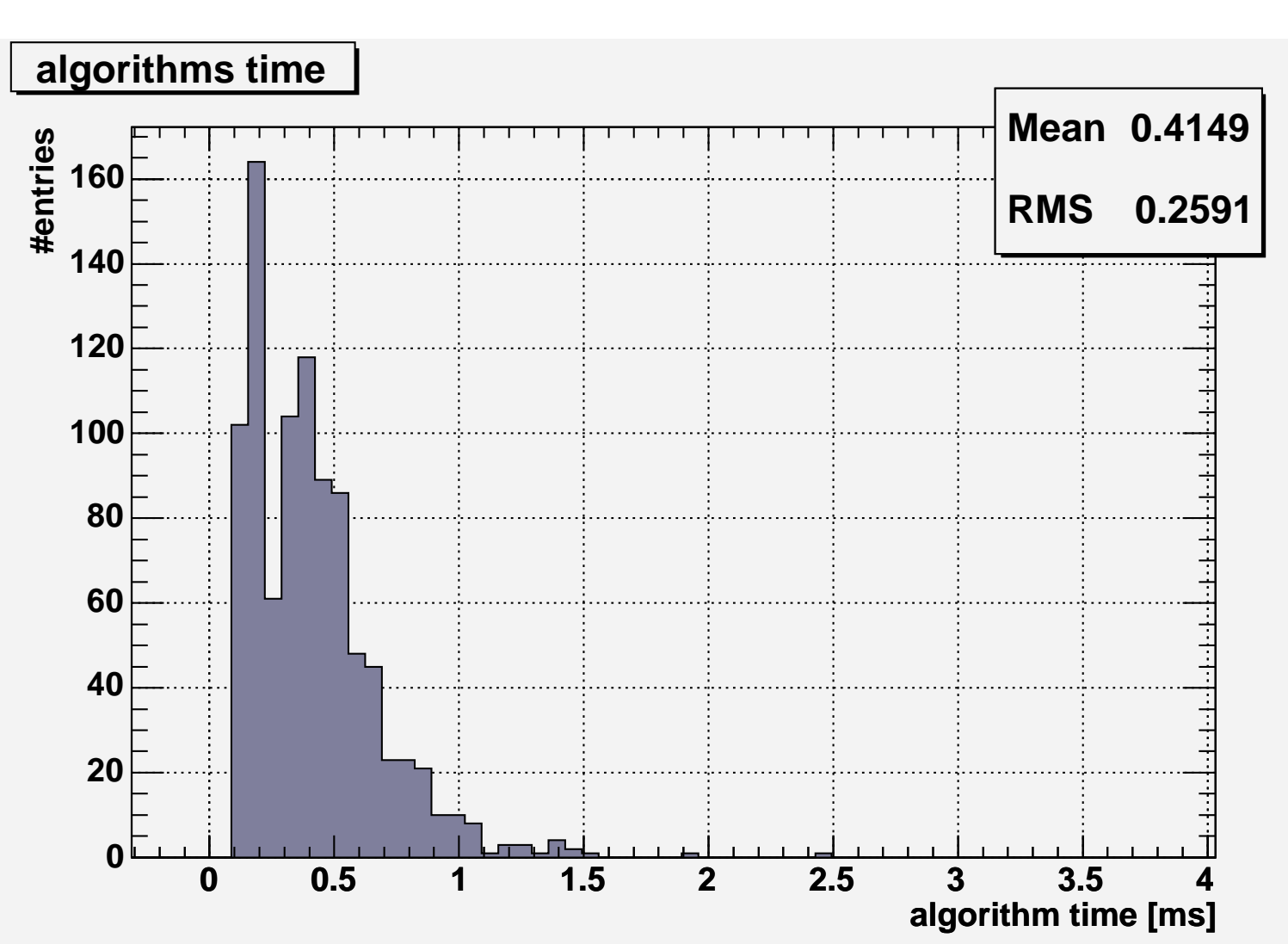

algorithms time 